\title{
Portomesenteric vein thrombosis following laparoscopic sleeve gastrectomy in a Chinese patient
}

\author{
KM Kwok', FHKCP, FHKAM (Medicine), KL Lee ${ }^{1}$, FHKCP, FHKAM (Medicine), YS Poon ${ }^{1}$, FHKCP, FHKAM (Medicine), \\ SY Lam ${ }^{1}$, FHKCP, FHKAM (Medicine), T Liong1', FHKCP, FHKAM (Medicine), HM Wong'1, MBChB, MRCP, \\ NK Chiu ${ }^{2}$, MBChB, FHKAM (Surgery), KI Law ${ }^{1}$, FHKCP, FHKAM (Medicine)
}

Departments of ${ }^{1}$ Intensive Care and ${ }^{2}$ Surgery, United Christian Hospital, Kwun Tong, Hong Kong

*Corresponding author: lawki@ha.org.hk

Hong Kong Med J 2017;23:651-2

DOI: $10.12809 / \mathrm{hkmj} 166321$

\section{Case report}

A 51-year-old morbidly obese Chinese man was scheduled for laparoscopic sleeve gastrectomy in October 2016. He had a body mass index of $34 \mathrm{~kg} / \mathrm{m}^{2}$ complicated by metabolic syndrome. He had no history of thromboembolism. Surgery was performed using a 5-port technique. A liver retractor was inserted under direct vision. The greater curvature was mobilised up to the angle of His and the gastric sleeve was created. The operation lasted 125 minutes. The patient was mobile on postoperative day 2 and was discharged on day 5.

On day 6 postoperatively, he presented to the surgical ward with nausea, vomiting, and epigastric pain. No peritoneal signs were elicited during physical examination. White cell count had increased to $10.1 \times 10^{9} / \mathrm{L}$ (reference range, 4-11 x $10^{9} / \mathrm{L}$ ), and serum creatinine level to $248 \mu \mathrm{mol} / \mathrm{L}$ (reference range, 67-109 $\mu \mathrm{mol} / \mathrm{L}$ ). He was kept nil by mouth and prescribed broad-spectrum antibiotics. A computed tomographic (CT) scan of the abdomen and pelvis with intravenous contrast was performed on postoperative day 8 . The portal vein was not opacified and a wedge-shaped hypoenhancing area was seen at subcapsular S4 of the liver. These were attributed by the radiologist to the timing of acquisition and perfusion artefacts. Ascites was also identified on the CT scan.

His condition deteriorated and he was transferred to the intensive care unit on postoperative day 9. A repeat contrast-enhanced CT on the same day, arranged in view of his rapid deterioration and the presence of unexplained ascites, revealed extensive thrombosis of the superior mesenteric vein, splenic vein, portal trunk, and portal veins (Fig). A long segment of small bowel appeared ischaemic. Hypoenhancement in the liver and spleen was evident and likely related to impaired perfusion. Emergent laparotomy was performed immediately and revealed small bowel gangrene extending from the proximal jejunum to mid-ileum with mesenteric vein thrombosis. The distal ileum showed venous

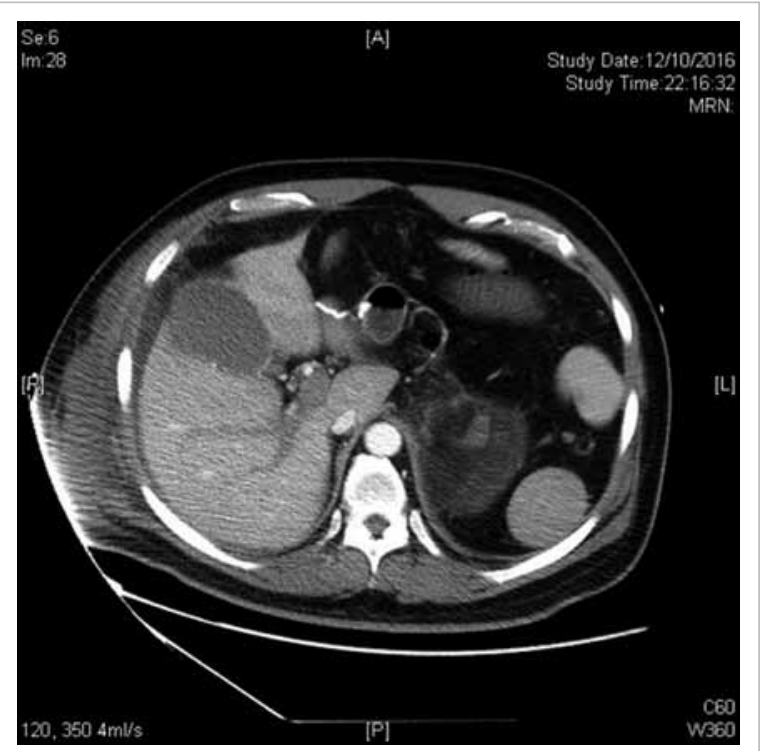

FIG. Computed tomography image showing portal vein thrombosis and hypoenhancement in the central part of liver

congestion. The surgical team attempted to perform clot retrieval by insertion of a Fogarty catheter to the ileal branch of the mesenteric vein but was unsuccessful due to the extensive thrombosis. A gangrenous segment of small bowel was then resected. The operation took 5 hours; the patient remained anuric and required escalating pressor support intra-operatively.

He was kept intubated and transferred back to the intensive care unit postoperatively. He had severe metabolic acidosis with arterial blood gas $\mathrm{pH}$ of 7.16 (reference range, 7.35-7.45). He required renal support by continuous venovenous haemofiltration from postoperative day 1 to 3 . Renal function later recovered and no further renal replacement therapy was needed. The patient was weaned off vasopressors on day 4 and was extubated on day 6 .

Due to the presence of coagulopathy, 
anticoagulation was not prescribed immediately postoperatively. Intravenous unfractionated heparin was introduced on postoperative day 3 with close monitoring of activated partial thromboplastin time. The infusion was withheld on day 6 as the patient passed malaena. Oesophagogastroduodenoscopy and colonoscopy did not reveal any sites of bleeding. Heparin infusion was then resumed and on day 8 changed to subcutaneous low-molecular-weight heparin.

The patient was discharged from the intensive care unit on postoperative day 12 . Closure of the ileostomy was performed in January 2017. Oral anticoagulation was prescribed for at least 6 months and follow-up CT scan was arranged to monitor the progress of portomesenteric vein thrombosis (PMVT)

\section{Discussion}

Portomesenteric vein thrombosis is an infrequent but potentially life-threatening complication following laparoscopic bariatric surgery. To the best of our knowledge, this is the first case report of PMVT as a complication of laparoscopic bariatric surgery in the Chinese population. Previous retrospective studies have reported an incidence of $0.3 \%$ to $1 \%,{ }^{1-3}$ although this was likely underestimated due to the presence of asymptomatic cases. Most patients run an indolent course and do not require any surgical intervention. Nonetheless some cases may be fulminant as in our patient with mesenteric ischaemia and infarction. ${ }^{1-5}$

The initial manifestation of PMVT can be subtle so early diagnosis requires a high index of suspicion. Patients usually present 7 to 14 days postoperatively with nausea, vomiting, abdominal pain, and fever. ${ }^{1,4,5}$ Physical examination is mostly unrevealing. Apart from leukocytosis and mild elevation of liver enzymes, most laboratory tests are normal. Only when it is associated with mesenteric ischaemia do patients present with peritonitis and septic shock. Initially, our patient presented typically with non-specific gastrointestinal upset but then deteriorated rapidly once bowel ischaemia occurred.

Various mechanisms of PMVT following laparoscopic bariatric surgery have been proposed. The use of a reverse Trendelenburg position and carbon dioxide pneumoperitoneum may cause a decrease in portal blood flow leading to stasis. ${ }^{1,4,5}$ The change in blood flow due to ligation of the short gastric vessels may promote the occurrence of PMVT ${ }^{1,2}$ Direct contact with the splenic vein during surgery may result in intimal damage and subsequent thrombosis. ${ }^{1,2,4}$ The use of a liver retractor can lead to blood stasis within the liver; retrograde thrombosis may occur. ${ }^{2}$ Finally, patients may have difficulty in maintaining adequate fluid intake following bariatric surgery. Dehydration will increase the risk of thrombotic complications. ${ }^{1,2}$

Contrast-enhanced $\mathrm{CT}$ scan is used to diagnose PMVT with a sensitivity of $90 \% .{ }^{2}$ Ascites is present in approximately one third of patients with PMVT. ${ }^{4}$ The presence of unexplained ascites following laparoscopic bariatric surgery should not be overlooked.

Treatment of PMVT depends on its severity. Therapeutic anticoagulation is recommended in patients without mesenteric ischaemia with an aim to recanalise the portomesenteric veins. ${ }^{6}$ Nonetheless the optimal duration of anticoagulation is not well defined. Gandhi et $\mathrm{al}^{7}$ suggested 3 to 6 months of anticoagulation, and extended further if signs and symptoms persist. Other studies have recommended longer treatment, ranging from 6 to 12 months., In cases with underlying thrombophilia, lifelong anticoagulation is required. Prompt anticoagulation can reduce the future risk of extrahepatic portal hypertension with the associated complications such as variceal gastrointestinal bleeding. ${ }^{2}$ In severe cases of PMVT complicated with bowel ischaemia, immediate exploration and bowel resection is mandated. Direct portomesenteric thrombectomy or thrombolysis is possible in selected cases. ${ }^{1}$

\section{References}

1. Goitein D, Matter I, Raziel A, et al. Portomesenteric thrombosis following laparoscopic bariatric surgery: incidence, patterns of clinical presentation, and etiology in a bariatric patient population. JAMA Surg 2013;148:3406.

2. Villagrán R, Smith G, Rodriguez W, et al. Portomesenteric vein thrombosis after laparoscopic sleeve gastrectomy: incidence, analysis and follow-up in 1236 consecutive cases. Obes Surg 2016;26:2555-61.

3. Salinas J, Barros D, Salgado N, et al. Portomesenteric vein thrombosis after laparoscopic sleeve gastrectomy. Surg Endosc 2014;28:1083-9.

4. Rosenberg JM, Tedesco M, Yao DC, Eisenberg D. Portal vein thrombosis following laparoscopic sleeve gastrectomy for morbid obesity. JSLS 2012;16:639-43.

5. Muneer M, Abdelrahman H, El-Menyar A, et al. Portomesenteric vein thrombosis after laparoscopic sleeve gastrectomy: 3 case reports and a literature review. Am J Case Rep 2016;17:241-7.

6. Condat B, Pessione F, Hillaire S, et al. Current outcome of portal vein thrombosis in adults: risk and benefit of anticoagulant therapy. Gastroenterology 2001;120:490-7.

7. Gandhi K, Singh P, Sharma M, Desai H, Nelson J, Kaul A. Mesenteric vein thrombosis after laparoscopic gastric sleeve procedure. J Thromb Thrombolysis 2010;30:179-83.

8. Kumar S, Sarr MG, Kamath PS. Mesenteric venous thrombosis. N Engl J Med 2001;345:1683-8. 